\title{
Enolase and dipeptidyl peptidase IV protein sub-unit vaccines are not protective against a lethal Streptococcus suis serotype 2 challenge in a mouse model of infection
}

Audrey Dumesnil ${ }^{1,2}$, Léa Martelet ${ }^{1,2}$, Daniel Grenier ${ }^{2,3}$, Jean-Philippe Auger ${ }^{1,2}$, Josée Harel ${ }^{1,2}$, Eric Nadeau ${ }^{4}$ and Marcelo Gottschalk ${ }^{1,2^{*}}$

\begin{abstract}
Background: Streptococcus suis is a major swine pathogen causing arthritis, meningitis and sudden death in postweaning piglets and is also a zoonotic agent. S. suis comprises 35 different serotypes of which the serotype 2 is the most prevalent in both pigs and humans. In the absence of commercial vaccines, bacterins (mostly autogenous), are used in the field, with controversial results. In the past years, the focus has turned towards the development of sub-unit vaccine candidates. However, published results are sometimes contradictory regarding the protective effect of a same candidate. Moreover, the adjuvant used may significantly influence the protective capacity of a given antigen. This study focused on two protective candidates, the dipeptidyl peptidase IV (DPPIV) and the enolase (SsEno). Both proteins are involved in S. suis pathogenesis, and while contradictory protection results have been obtained with SsEno in the past, no data on the protective capacity of DPPIV was available.

Results: Results showed that among all the field strains tested, 86 and $88 \%$ were positive for the expression of the SsEno and DPPIV proteins, respectively, suggesting that they are widely expressed by strains of different serotypes. However, no protection was obtained after two vaccine doses in a CD-1 mouse model of infection, regardless of the use of four different adjuvants. Even though no protection was obtained, significant amounts of antibodies were produced against both antigens, and this regardless of the adjuvant used.

Conclusions: Taken together, these results demonstrate that S. suis DPPIV and SsEno are probably not good vaccine candidates, at least not in the conditions evaluated in this study. Further studies in the natural host (pig) should still be carried out. Moreover, this work highlights the importance of confirming results obtained by different research groups.
\end{abstract}

Keywords: Streptococcus suis serotype 2, Sub-unit vaccines, Antibody production, Protection, Mouse model, Experimental design, bias

\footnotetext{
* Correspondence: marcelo.gottschalk@umontreal.ca

${ }^{1}$ Groupe de recherche sur les maladies infectieuses en production animale (GREMIP), Faculty of Veterinary Medicine, University of Montreal, 3200 Sicotte St., Saint-Hyacinthe, QC J2S 2M2, Canada

${ }^{2}$ Swine and Poultry Infectious Diseases Research Center (CRIPA), Montreal,

Quebec, Canada

Full list of author information is available at the end of the article
}

(c) The Author(s). 2019 Open Access This article is distributed under the terms of the Creative Commons Attribution 4.0 International License (http://creativecommons.org/licenses/by/4.0/), which permits unrestricted use, distribution, and reproduction in any medium, provided you give appropriate credit to the original author(s) and the source, provide a link to the Creative Commons license, and indicate if changes were made. The Creative Commons Public Domain Dedication waiver (http://creativecommons.org/publicdomain/zero/1.0/) applies to the data made available in this article, unless otherwise stated. 


\section{Background}

Streptococcus suis (S. suis) is one of the most frequent causes of mortality in weaned piglets worldwide, causing mainly septicemia with sudden death, meningitis and arthritis (1). It is also considered an emerging zoonotic agent, mainly in South-East Asia, as an etiological agent of meningitis and septic shock $(2,3)$. S. suis is classified into 35 serotypes based on the antigenicity of the capsular polysaccharide (CPS). More recently, serotypes $20,22,26,32,33$, and 34 have been suggested to belong to different bacterial species (4), whereas strains with new CPS genes have also been described (5). Serotype 2 is reported as being the most virulent and frequently recovered serotype from diseased animals (6). However, other serotypes have also been described to be able to cause serious diseases, mainly 5, 7, 9 and 14 (7). In humans, serotype 2 is also by far the serotype most frequently recovered from ill patients, followed by serotype 14 (6).

Early steps of the $S$. suis infection mainly take place in the upper respiratory and, as more recently suggested, the intestinal tract, where bacteria adhere to and, to a certain extent, invade epithelial cells (8). Although the mechanisms are not completely understood, S. suis eventually reaches the bloodstream, remains extracellular by resisting phagocytosis, and causes disease (9). S. suis resistance to phagocytosis by professional phagocytes is mainly due to the presence of the CPS (9). It is not unusual to have more than one serotype (and sometimes, different strains of the same serotype) involved in clinical cases in a given herd (3).

Early medicated weaning and segregated early weaning practices do not eliminate S. suis infection (3). Therefore, effective control measures to prevent disease depend on control of predisposing factors, prophylactic/ metaphylactic procedures (where allowed) and/or vaccination (3). Field reports describing vaccine failure are common $(10,11)$. Indeed, commercial vaccines are almost inexistent and those used in the field are mostly autogenous bacterins (10). With some exceptions, a limited protective response is usually reported with bacterins, which may be attributed to failure of the whole-bacterial antigens to elicit an immune response. This defective immunogenicity may be due, at least in part, to the presence of a low immunogenic CPS, to the loss of antigenicity caused by heat or formalin processing, production of antibodies to antigens not associated with protection, serotype-specific protection (when different serotypes are inducing disease in a given herd), and/or other unknown reasons (10).

Most research studies on $S$. suis vaccines have been performed with sub-unit candidates, which are based on proteins, with the exception of a serotype-specific CPS-conjugate vaccine (12). The main objective of protein-based sub-unit vaccines is usually to obtain a highly immunogenic cross-reactive antigen that would eventually protect against different serotypes (and strains) of S. suis. Indeed, more than 40 protein candidates have been shown to induce either protective antibodies (through in vitro opsonophagocytosis tests) or protection after in vivo challenge, mostly with serotype 2 strains $(10,13-16)$. With the exception of the suilysin and Sao proteins, which have been reported to be relatively constant in protection (with a few exceptions) when tested by independent research groups $(10,17)$, other vaccine candidates either presented contradictory results or were never tested by independent research groups (10). To further complicate the interpretation of results, it has been demonstrated that a given protein may either be protective or non-protective depending on the adjuvant used $(10,18,19)$.

In the present study, two sub-unit vaccine candidates, the dipeptidyl peptidase IV (DPPIV) and the enolase (SsEno), which are both membrane-associated proteins, were tested in a mouse model of infection in combination with one of four different adjuvants. The DPPIV, although not critical for virulence, has been described to play important functions in the pathogenesis of the infection caused by S. suis (20), but has never been tested as a vaccine candidate. The SsEno is a protein that has been described as playing important roles as a virulence factor $(21,22)$, but presented contradictory results when used in vaccination trials $(23,24)$. The presence of such vaccine protein candidates in a large collection of field strains of $S$. suis belonging to different serotypes has also never been tested.

\section{Results}

Production of DPPIV and SsEno by field strains of S. suis belonging to different serotypes

Antisera produced against recombinant enolase ( $\mathrm{rSsEno}$ ) and DPPIV (rDPPIV) showed clear reactions with purified proteins of expected molecular masses of approximately $75 \mathrm{kDa}$ and $100 \mathrm{kDa}$, respectively (Fig. $1 \mathrm{~A}$ and B). The constitutional expression of SsEno and DPPIV was then evaluated in $S$. suis field strains by dot-blot with these mono-specific polyclonal hyperimmune rabbit sera. At least one of the two proteins were found in all $S$. suis serotypes, with the exception of the only serotype 6 strain available (Table 2). In the case of SsEno, $86 \%$ of all field strains belonging to all other serotypes were recognized by the anti sera against the rSsEno. Moreover, the DPPIV protein was expressed by $88 \%$ of the field strains tested belonging to all serotypes, with the exception of serotype 6 (as mentioned above), and the only available serotype 32 strain. 


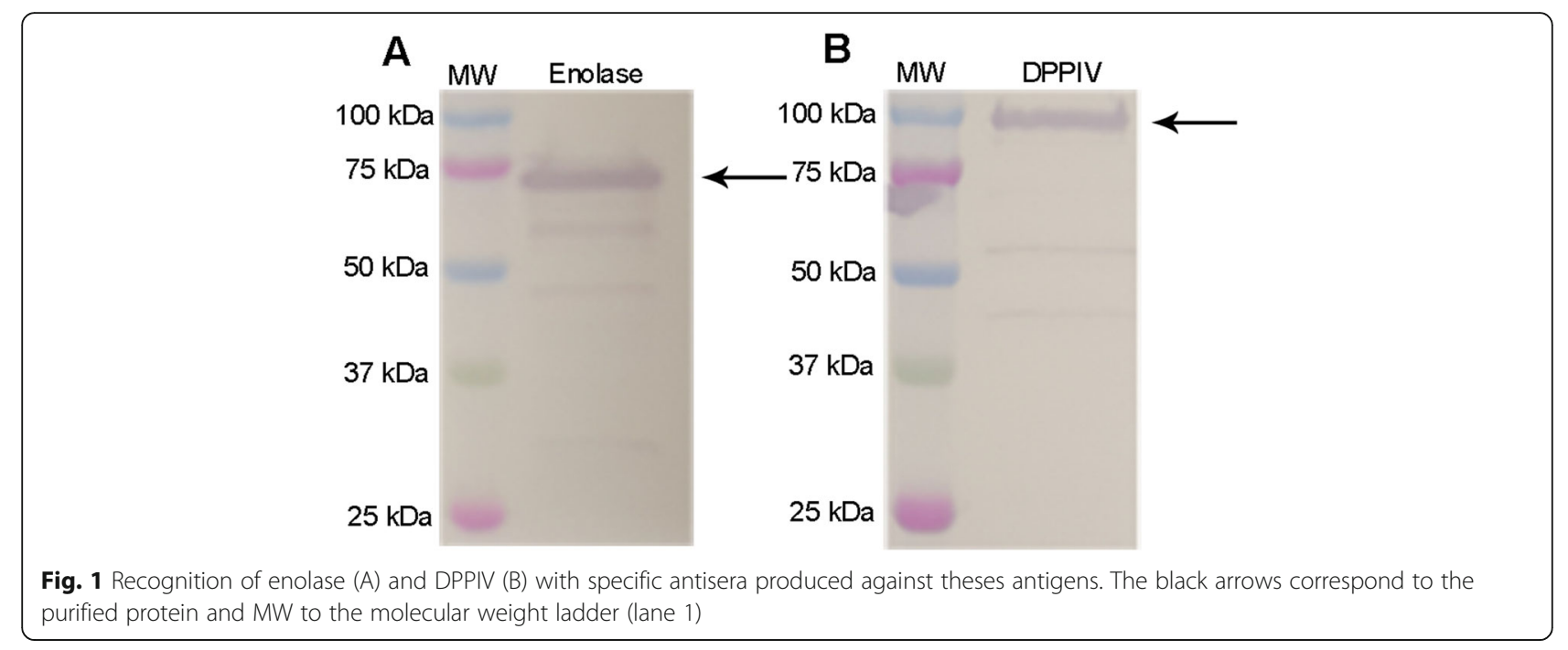

\section{Protection assays}

Twenty-four hours after challenge infection with the 31533 strain of S. suis serotype 2, mice from all groups began to present severe clinical signs of disease including rough hair coat, depression, prostration, and general weakness, leading to the euthanasia of certain individuals. Mortality during the first 5 days was mostly due to septicemia with bacterial colonization of systemic organs (data not shown). However, from day 6 post-challenge, several of the mice developed severe signs of central nervous system infection including pedaling, running in circles and opisthotonos. At least 8/11 mice immunized with rSsEno (independently of the adjuvant used) died or were euthanatized for ethical reasons at the end of the experiment (14 days post-infection) (Fig. 2A). In addition, less than $7 / 13$ animals immunized with the rDPPIV and the different adjuvants survived 14 days post-infection (Fig. 2B). Indeed, at the end of the trial, no significant differences in survival between the control group and any of the immunized animals (either with rSsEno or rDPPIV) could be observed $(p>0.05)$.

\section{Evaluation of the antibody response}

The efficacy of four different adjuvants in combination with either rSsEno or rDPPIV was compared regarding their capacity to induce the production of specific antibodies in a mouse model of infection. The production of specific antibodies against rSsEno and rDPPIV was evaluated before and after the first and second (boost) vaccination (Figs. 3 and 4). Overall, the four different adjuvants induced high total Ig titers $(\operatorname{IgG}+\operatorname{IgM})$ for both antigens after both vaccination doses $(p<0.05)$. Isotype switching (IgG1, IgG2b, IgG2c, and IgG3) was also observed for both antigens in the different combinations. However, the use of Stimune gave a stronger antibody response against $\mathrm{rSsEno}$ after the first vaccination in the case of IgG1 or the booster for IgG2b in comparison to both Quil- $\mathrm{A}^{\circ}$ and Polygen ${ }^{\mathrm{Tm}}(p<0.05)$, but not in comparison with Montanide ${ }^{\mathrm{Tm}}$ ISA $50 \mathrm{~V} 2$. Stimune ${ }^{\bullet}$ was also able to induce a strong antibody response against rDPPIV after the first vaccination dose in the case of IgG3 compared to Quil- $\mathrm{A}^{\circ}(\mathrm{p}<0.05)$ or the booster vaccination in the case of IgG1 and IgG2b in comparison with Quil- $\mathrm{A}^{\circ}$ and Polygen ${ }^{\mathrm{mm}}$ or Quil- $\mathrm{A}^{\bullet}$ and Montanide ${ }^{\mathrm{mm}}$ ISA 50 V2, respectively $(p<0.05)$. Polygen ${ }^{\mathrm{Tm}}$ enhanced IgG2c antibodies against rDPPIV after the first dose of the vaccine, compared to Quil- $\mathrm{A}^{\circ}$ and Montanide ${ }^{\mathrm{ma}}$ ISA 50 V2. However, Montanide ${ }^{\mathrm{Tm}}$ ISA 50 V2 induced a stronger IgG1 response against rDPPIV than Quil- $\mathrm{A}^{\circ}$ for both vaccinations, as well as Polygen ${ }^{\mathrm{Tm}}$ for the first vaccination only $(p<0.05)$. For both antigens, IgM production was lower than IgG production and a lighter booster effect was induced after the second vaccination, with the exception of Stimune ${ }^{\circ}$ with rSsEno. However, Stimune was, once again, the adjuvant giving one of the strongest IgM responses for both antigens $(p<0.05)$, even if there was no booster effect in combination with rSsEno. In general, and for both antigens, a relatively homogenous response was observed for IgM, IgG1 and IgG2b, but a higher heterogeneity was observed for IgG2c and IgG3. Finally, as expected, no detectable antibody titers against the proteins were observed in nonimmunized mice (Figs. 3 and 4).

\section{Discussion}

S. suis is considered one the most important swine bacterial pathogens in North America (25) and causes important economic losses to the swine industry worldwide (25). Control of S. suis disease is frustrating and complicated (10). Antibiotics can prevent clinical outbreaks, but the most effective belong to categories whose use in the swine industry has been greatly reduced given 

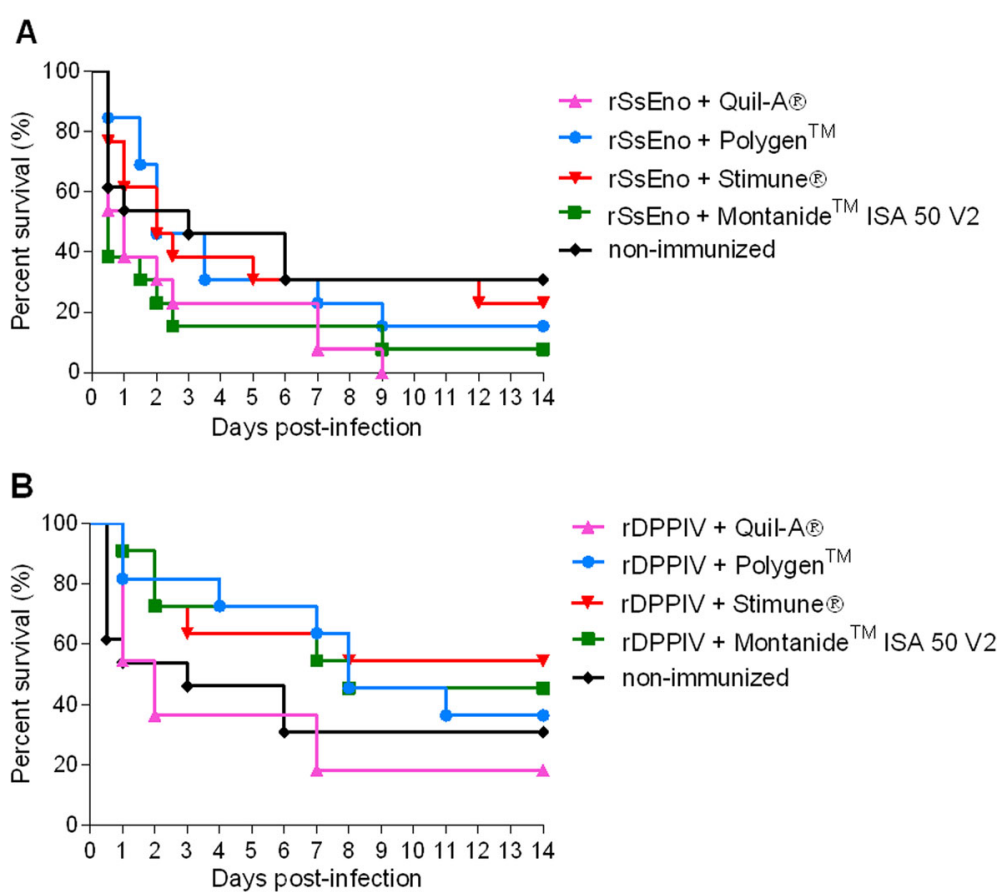

Fig. 2 The combination of rSsEno or rDPPIV with 4 different adjuvants is not protective against S. suis. Survival of the mice immunized with $50 \mu \mathrm{g}$ of rSsEno (A) or rDPPIV (B) with either Polygen ${ }^{\mathrm{TM}}$, Montanide ${ }^{\mathrm{TM}}$ ISA 50 V2, Quil-A ${ }^{\oplus}$ or Stimune ${ }^{\oplus}$ adjuvants following challenge with S. suis 31533 strain. A control group (non-immunized) received $100 \mu \mathrm{L}$ of phosphate-buffered saline

their importance in both human and veterinary medicine (26). In addition, the prophylactic and methaphylactic use of antibiotics in swine has been reduced or banished in several European countries. Control of stress factors, such as overcrowding, poor ventilation, high humidity, and inadequate sanitation, as well as the control of concomitant diseases (such as Porcine Reproductive and Respiratory Syndrome Virus), should be part of a global strategy to prevent clinical cases (25). However, in the presence of high virulent strains, protective vaccines are necessary.

The high heterogeneity of serotypes (and strains within a single serotype) has so far precluded the use of universal vaccines. Indeed, autogenous bacterins are mostly used in the field with questioned protection. Many studies in the last years have focused on the identification of cross-reacting proteins with protective capacities (10). Ideally, these proteins must be highly immunogenic and present in most field strains. In the present study, two proteins have been evaluated as such immunogens, whether the enolase and the DPPIV. In addition to its conserved functions in carbohydrate metabolism, the enolase has been suggested to be a virulence factor due to its capacity to bind to fibronectin, plasminogen and laminin, to enhance invasion of the blood-brain barrier and to induce pro-inflammatory cytokine production in the central nervous system $(21,22)$. In addition, the enolase has been shown to be protective for other pathogenic streptococci $(27-29)$. In the present study, we showed that more than $85 \%$ of the strains tested reacted with a mono-specific antibody against rSsEno, which indicates that this protein is widely expressed by $S$. suis strains. Being an important proposed virulence factor and since it is widely present in field strains, the enolase would make an interesting protective candidate. However, results obtained so far are contradictory, showing either no or a relatively good protection by two different research groups $(23,24)$. In the present study, antibody production and protection in a mouse model of infection have been studied. Since it has been clearly proposed that the adjuvant used may influence the protection (and different IgG isotypes) obtained with a $S$. suis vaccine, we also tested four different adjuvants. Although small differences were observed, antibody response was generally high with all adjuvants, with a good response after the first dose and a booster response after the second vaccine dose. Whereas a strong IgG1 and IgG2b response was observed, a relatively lower and less homogenous response was observed for IgG2c and IgG3, confirming previous results obtained with other proteins (18). Independently of the antibody titer, no significant protection was observed with any of the 


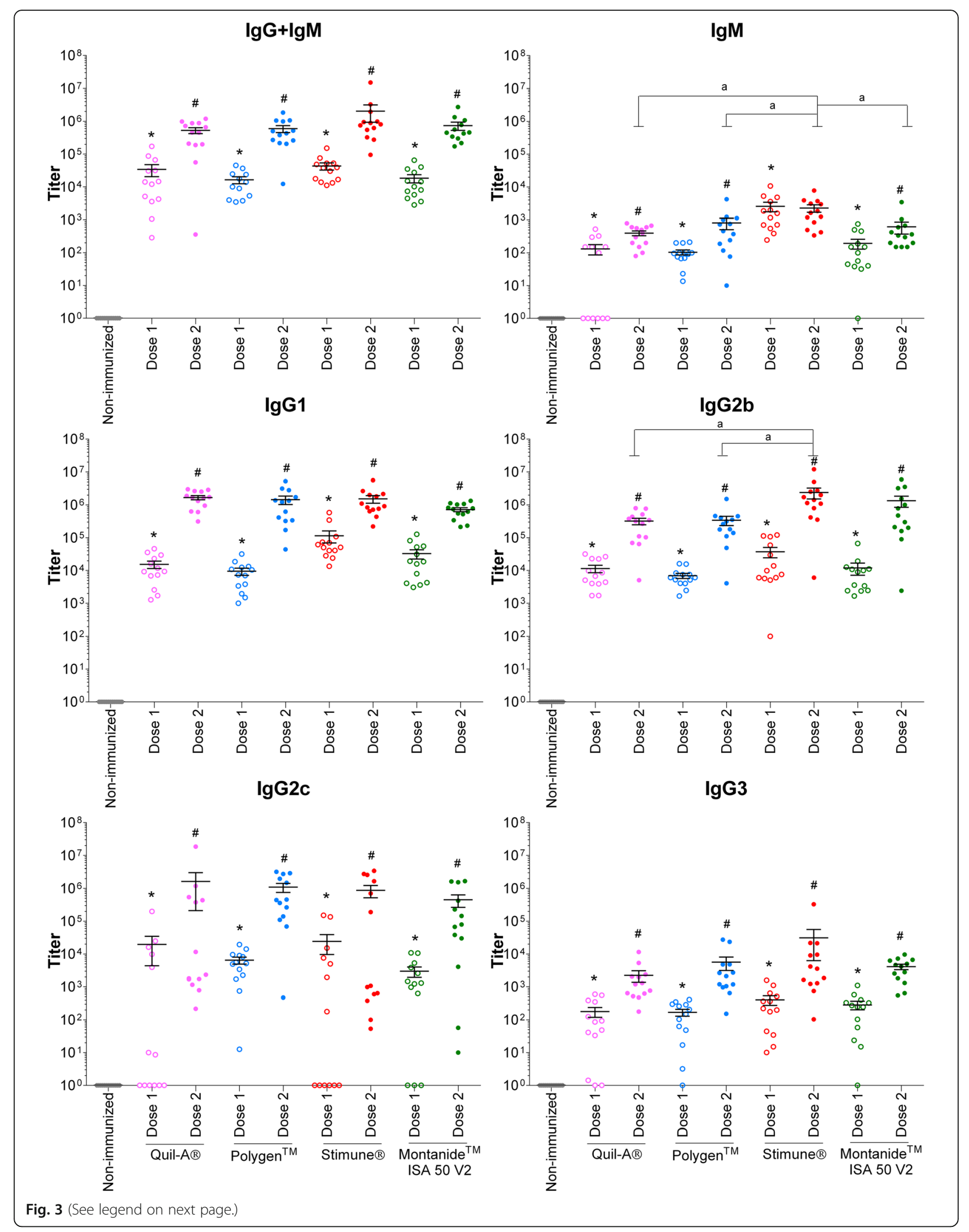


(See figure on previous page.)

Fig. 3 Titers of anti-Enolase antibody isotypes of mice immunized with rEnolase in combination with 4 adjuvants. Two $50 \mu g$ doses of rSsEno adjuvanted in Quil-A ${ }^{\oplus}$, Polygen ${ }^{T M}$, Stimune ${ }^{\oplus}$ or Montanide ${ }^{T M}$ ISA 50 V2 were administered to mice. A control group (non-immunized) received $100 \mu \mathrm{L}$ of phosphate-buffered saline. Isotypes were detected in sera twelve days after the first (Dose 1) and the second (Dose 2) vaccination. Data are expressed as mean \pm standard error of the mean. ${ }^{*}(p<0.05)$ indicates a significant difference between non-immunized and dose 1 . \# $(p<$ 0.05 ) indicates a significant difference between dose 1 and dose 2 and $a(p<0.05)$ between treated groups (adjuvants) for the second immunization (Dose 2)

adjuvants used. These results confirm those from Esgleas et al. (23), but are in contradiction with those obtained previously $(24,30)$. It is important to mention that the rSsEno was cloned from strain P1/7 strain in this study and that from Esgleas et al. (23), whereas strain 05ZYH33 was used in the successful protective studies of both Zhang et al. and Feng et al. $(24,30)$. There is $94 \%$ identity between P1/7 and 05ZYH33 enolase genes and in both cases, the complete gene was cloned into expression vectors. In the present study, the plasmid pET-32a used carries a C-terminal his-tag, while the plasmid pET28a used by Zhang et al carries two his-tags (C- and N-terminal) (24). In addition, an infection model of either inbreeding $\mathrm{BALB} / \mathrm{c}(24,30)$ or outbred $\mathrm{CD}-1$ mice (Esgleas et al. (23) and the present study), were used. It is not possible to ascertain if such differences may be responsible for the discrepancy in the protective capacity of this recombinant protein. The use of inbred mice may influence the immune response of such animals to different pathogens (31). For example, $\mathrm{BALB} / \mathrm{c}$ mice tend toward a Th2-predominant response (32). Indeed, outbred lines of mice are a better model to evaluate protection since they better represent the natural population (33).

The dipeptidyl aminopeptidase IV (DPPIV) is a serine protease that cleaves X-Pro/Ala di-peptide from the Nterminus of proteins and is present in S. suis (34). It has been recently demonstrated to bind fibronectin (35), although its role as a critical virulence factor is controversial $(20,35,36)$. Interestingly, it has never been tested as a potential vaccine candidate. As was the case with the enolase, the DPPIV is largely present in S. suis field strains, which makes it an interesting vaccine candidate. However, results obtained in the present study are very similar to those obtained with the enolase: high antibody titers and good isotype switching, but lack of protection with the four adjuvants tested.

Finally, it should be considered that, although no protection with individual proteins were observed in the present study, it might be interesting to test if a combination of both sub-unit proteins with an appropriate adjuvant may induce some kind of protection. In addition, final confirmation using the natural host, the pig, would also be interesting. However, it should considered that so far, no single protein has been described as being non-protective in mice and protective in pigs.

\section{Conclusion}

Although both proteins are present in most tested S. suis isolates, the recombinant proteins obtained in the present study did not confer protection with any of the adjuvant tested in an outbred model of infection despite the presence of specific IgM and different IgGs subtypes. These results confirm the difficulties in obtaining and evaluating (with good reproducibility) protective sub-unit protein candidates to control S. suis infections. Differences in the characteristics of the recombinant proteins produced, adjuvant used, animal model (animal species, inbred vs outbred), and S. suis strain used may significantly modify the obtained results. This problematic reinforces the concept and need of confirming published results by different research groups, as recently described for the role of critical virulence factors (37).

\section{Methods}

\section{Bacterial strains and growth conditions}

All strains and plasmids used in this study are listed in Table 1 . The S. suis serotype 2, strain 31533, isolated from a pig with meningitis and previously used in protection studies, was chosen for challenging mice (38). The expression of SsENo and DPPIV proteins at the bacterial surface was evaluated by dot-blot. This strain was cultured as previously described with a few modifications (39). Briefly, bacteria were grown overnight onto sheep blood agar plates at $37^{\circ} \mathrm{C}$ and isolated colonies were cultured in $5 \mathrm{~mL}$ of Todd-Hewitt broth (THB; Becton Dickinson, Mississauga, ON, Canada) for $8 \mathrm{~h}$ at $37^{\circ} \mathrm{C}$. Then, $10 \mu \mathrm{L}$ of a $10^{-3}$ dilution of $8 \mathrm{~h}$-cultures were transferred into $30 \mathrm{~mL}$ of THB and incubated for $16 \mathrm{~h}$ at $37^{\circ} \mathrm{C}$. Stationary phase bacteria were washed in phosphate-buffered saline (PBS, pH 7.3). The bacterial pellet was then resuspended in THB and adjusted to the desired concentrations. Escherichia coli was grown in Luria-Bertani medium (Becton-Dickinson) containing $100 \mu \mathrm{g} / \mathrm{mL}$ of ampicillin (Sigma-Aldrich, Oakville, ON, Canada) when needed (38).

A total of 359 S. suis field strains, belonging to different serotypes (Table 2), were tested for the expression of SsEno and DPPIV proteins as described below. These 


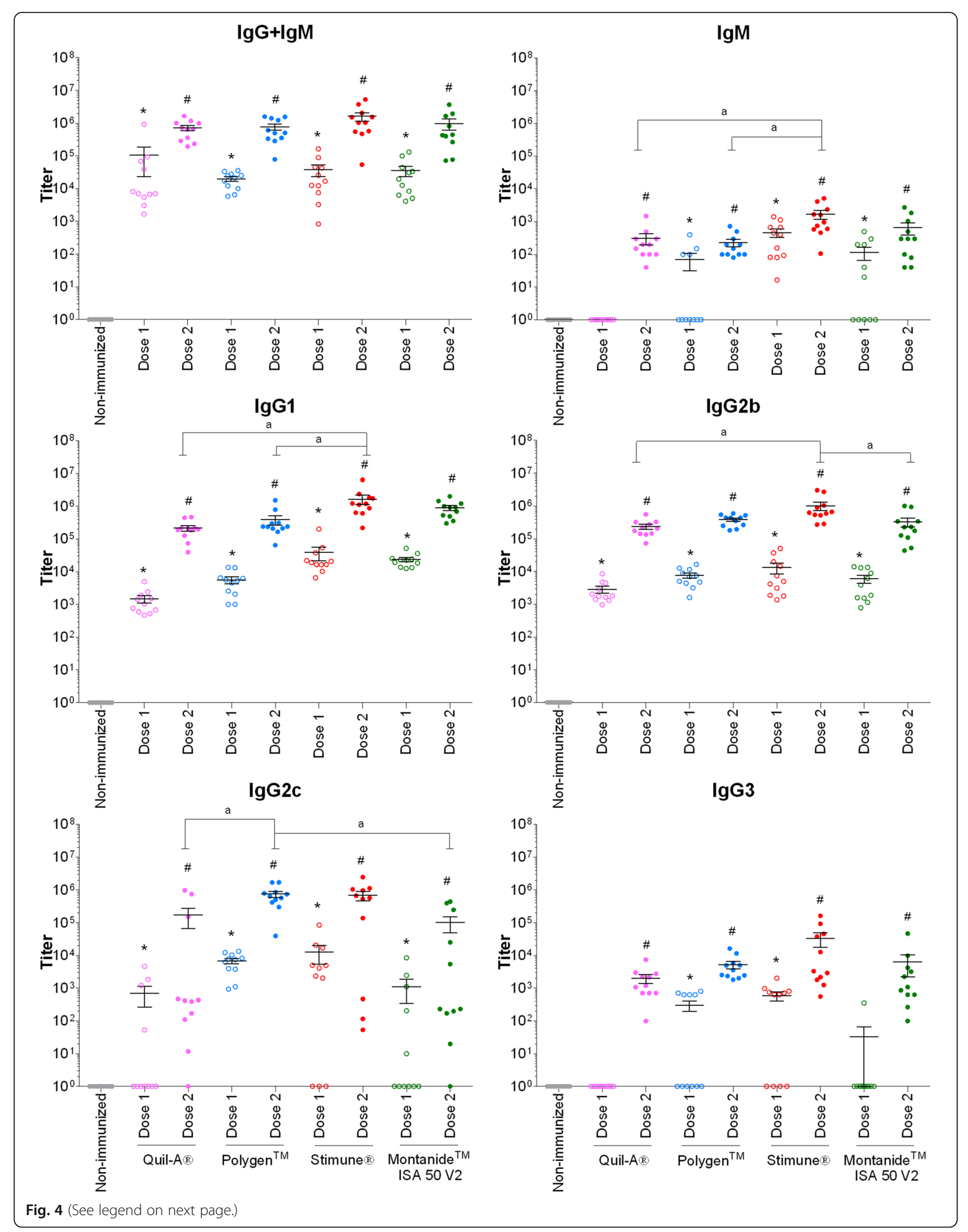


(See figure on previous page.)

Fig. 4 Titers of anti-DPPIV antibody isotypes of mice immunized with rDPPIV in combination with 4 adjuvants. Two $50 \mu g$ doses of rDPPIV adjuvanted in Quil-A ${ }^{\oplus}$, Polygen ${ }^{\text {TM }}$, Stimune ${ }^{\oplus}$ or Montanide ${ }^{\text {TM }}$ ISA 50 V2 were administered to mice. A control group (non-immunized) received $100 \mu \mathrm{L}$ of phosphate-buffered saline. Isotypes were detected in sera twelve days after the first (Dose 1) and the second (Dose 2) vaccination. Data are expressed as mean \pm standard error of the mean. ${ }^{*}(p<0.05)$ indicates a significant difference between non-immunized and dose $1 . \#(p<$ 0.05 ) indicates a significant difference between dose 1 and dose 2 and $a(p<0.05)$ between treated groups (adjuvants) for the second immunization (Dose 2)

strains were isolated from the internal organs of diseased pigs in Canada and sent to the diagnostic laboratory of the Faculty of Veterinary Medicine of the University of Montreal. Bacteria were grown overnight onto sheep blood agar plates at $37^{\circ} \mathrm{C}$ and isolated colonies were cultured under agitation in $5 \mathrm{~mL}$ of THB for $16 \mathrm{~h}$ at $37^{\circ} \mathrm{C}$ at $120 \mathrm{rpm}$.

\section{Cloning, expression and purification of rSsEno and rDPPIV proteins}

The cloning and purification of the protein candidates were performed based on the methodology previously described $(21,34)$. Briefly, the sequence used to design primers for PCR amplification of the SsEno gene was the SsEno1-forward primer 5'-TATAAGGATCCTATAAG GATCCTTGTCAATTATTACTGATGTTTACGC-3', introducing a BamHI site (bold and underlined letters), and the Eno2-reverse primer 5'-TATAAAGCTTTTATTTTTTCAAGTTGTAGAATGAGTTCAAGCC-3', introducing a HindIII site (bold and underlined letters). The PCR amplified gene was confirmed by automated sequencing and cloned into pET-32a vector (Novagen, Madison, WI, USA), using the BamHI and HindIII sites. The plasmid pET-32aSsEno was transformed into E. coli Bl21DE3 for IPTG-inducible expression of recombinant
SsEno. The protein was purified by affinity chromatography using the His-Bind Resin Chromatography Kit (Novagen, Madison, WI, USA) according to manufacturer's instructions. In the case of the DPPIV, the selected gene was amplified by PCR using the following primers: 5' CGCTTTAATCAATTTTCTTTCATAAA AAAAGAGAC $3^{\prime}$ and $5^{\prime}$ TTTGGATTTTCATTGAGT ATTAGTGCG 3' and stop codons in concordance with the pBAD/thio-TOPO system (Invitrogen, Carlsbad, CA, USA). Cytosol extract were then purified in two steps, whether a Mono-Q 5/50 GL (GE Healthcare, Baie d'Urfé, Quebec, Canada) followed by a concentration with Superdex 200 10/300 GL (GE Healthcare), according to manufacturer's instructions. Previous data clearly showed that this technique lead to higher yields than using a $\mathrm{Ni}^{2+}$-nitrilotriacetic acid affinity chromatography column.

For both vaccine candidates, protein-containing fractions were determined by SDS-PAGE and Western blot using an anti-His-tag antibody (R\&D Systems, Minneapolis, MN, USA) and were dialysed. The purified recombinant proteins were then concentrated using Amicon Ultra-15 (Millipore, Billerica, MA, USA) and protein quantification was evaluated using the Pierce Bicinchoninic Acid Protein Assay Kit (Fisher Scientific, Ottawa,

Table 1 Bacterial strains and plasmids used in this study

\begin{tabular}{|c|c|c|}
\hline Strains/plasmids & General characteristics & $\begin{array}{l}\text { Source/ } \\
\text { reference }\end{array}$ \\
\hline \multicolumn{3}{|l|}{ Escherichia coli } \\
\hline TOP10 & $\begin{array}{l}\mathrm{F}^{-} \text {mrcA } \Delta \text { (mrr-hsdRMS-mcrBC) } \varphi 80 \text { lacZ } \Delta \text { M15 } \Delta \text { lacX74 recA1 araD139 } \Delta \text { (ara-leu) } 7697 \text { galU galK rpsL }\left(\text { Str }^{\mathrm{R}} \text { ) }\right. \\
\text { endA1 nupG }\end{array}$ & Invitrogen \\
\hline BL21 & $\mathrm{F}^{-}$ompT hsdS $\left(\mathrm{r}_{B}{ }^{-}, \mathrm{m}_{\mathrm{B}}{ }^{-}\right) \mathrm{gal} \mathrm{dcm}$ rne131 (DE3) & Invitrogen \\
\hline \multicolumn{3}{|l|}{ Streptococcus suis } \\
\hline 31533 & Virulent European serotype 2 ST1 strain isolated from pig with meningitis & $(42)$ \\
\hline$\Delta d p p / V$ & Isogenic mutant strain derived from $\mathrm{P} 1 / 7$ strain. In frame deletion of $d p p / V$ & (20) \\
\hline \multicolumn{3}{|c|}{ Group B Streptococcus } \\
\hline $\mathrm{COH}-1$ & Capsular type III strain isolated from an infant with bacteremia & $(40)$ \\
\hline \multicolumn{3}{|l|}{ Plasmids } \\
\hline pET-32a & $\mathrm{Ap}^{\mathrm{r}}, \mathrm{pBR} 322$ ori, $\mathrm{T7}$ promotor & Novagen \\
\hline pBAD/thio & $A p^{r}$, pUC ori, $P_{B A D}$ promotor & Invitrogen \\
\hline $\begin{array}{l}\text { pET- } \\
\text { 32aEnolase }\end{array}$ & pET-32a carrying eno gene for protein production & This work \\
\hline pBAD/thioDPPIV & pBAD/thio-TOPO carrying dppIV gene for protein production & This work \\
\hline
\end{tabular}


Table 2 Distribution of enolase and DPPIV among S. suis field strains

\begin{tabular}{|c|c|c|c|}
\hline Serotype & Number of strains & Number of positive strains for Enolase & Number of positive strains for DPPIV \\
\hline 1 & 23 & 23 & 23 \\
\hline $1 / 2$ & 30 & 26 & 29 \\
\hline 2 & 93 & 73 & 80 \\
\hline 3 & 18 & 14 & 14 \\
\hline 4 & 21 & 20 & 18 \\
\hline 5 & 7 & 5 & 4 \\
\hline 6 & 1 & 0 & 0 \\
\hline 7 & 18 & 15 & 16 \\
\hline 8 & 17 & 17 & 16 \\
\hline 9 & 31 & 26 & 30 \\
\hline 10 & 3 & 3 & 2 \\
\hline 13 & 1 & 1 & 1 \\
\hline 14 & 13 & 12 & 11 \\
\hline 15 & 1 & 1 & 1 \\
\hline 16 & 9 & 8 & 7 \\
\hline 17 & 2 & 2 & 1 \\
\hline 18 & 3 & 3 & 3 \\
\hline 19 & 3 & 3 & 2 \\
\hline 20 & 1 & 1 & 1 \\
\hline 21 & 5 & 4 & 4 \\
\hline 22 & 15 & 13 & 13 \\
\hline 23 & 10 & 10 & 10 \\
\hline 24 & 3 & 3 & 2 \\
\hline 25 & 1 & 1 & 1 \\
\hline 27 & 3 & 3 & 3 \\
\hline 28 & 7 & 6 & 7 \\
\hline 29 & 4 & 3 & 4 \\
\hline 30 & 4 & 4 & 4 \\
\hline 31 & 3 & 2 & 2 \\
\hline 32 & 1 & 1 & 0 \\
\hline 33 & 4 & 4 & 2 \\
\hline 34 & 4 & 3 & 4 \\
\hline
\end{tabular}

ON, Canada). Two mono-specific polyclonal hyperimmune sera were then produced in rabbits using the purified rSsEno and rDPPIV protein, respectively, and tested by immunoblot using the purified proteins as previously described (19).

\section{Detection of naturally expressed SsENo and DPPIV} proteins on the surface of $S$. suis field strains

Centrifugation of $5 \mathrm{mLS}$. suis overnight cultures in THB was performed and bacterial pellets were resuspended in PBS before treatment with $0.5 \%$ formaldehyde for $1 \mathrm{~h}$ at $37^{\circ} \mathrm{C}$. Ten $\mu \mathrm{l}$ of a formalin-killed whole bacteria suspension were blotted on a Nitrocellulose
Western blotting membrane (Bio-Rad, Hercules, CA, USA). The membrane was blocked for $1 \mathrm{~h}$ with a solution of Tris-buffered saline (TBS) containing $2 \%$ casein, followed by $2 \mathrm{~h}$ incubation with mono-specific polyclonal hyperimmune rabbit serum against either SsEno or DPPIV, diluted in blocking buffer. The membrane was then washed with TBS and further incubated for 1 $h$ with a goat anti-rabbit IgG horseradish peroxidase (HRP)-conjugated antibody (Jackson ImmunoResearch, West Grove, PA). The membrane was rinsed with TBS and revealed using a 4-chloro-1-naphthol solution (Sigma-Aldrich). The S. suis 31533 strain was used as a positive control. Group B Streptococcus serotype III 
$\mathrm{COH}-1$ strain (40) and a S. suis isogenic mutant defective in the production of DPPIV (20) were used as negative controls for SsEno and DPPIV detection, respectively.

\section{Immunization and protection studies}

All experiments involving mice were conducted in accordance with the guidelines and policies of the Canadian Council on Animal Care and the principles set forth in the Guide for the Care and Use of Laboratory Animals by the Animal Welfare Committee of the University of Montreal (RECH-1748). Housing and husbandry of animals were taken care by the personal of level II facilities at the University of Montreal. Six-weekold CD-1 mice (Charles River Laboratories, SaintConstant, QC, Canada) were randomly assigned to five groups of 11 or 13 mice for immunization with rSsEno or rDPPIV, respectively, as accepted by the ethical committee. Animals were immunized twice subcutaneously at a 2-week interval with either $50 \mu \mathrm{g}$ of $\mathrm{rSsEno}$ or rDPPIV mixed with one of the following adjuvants: $20 \mu \mathrm{g}$ of Quil-A (Brenntag Biosector, Frederikssund, Danemark), $15 \%$ of Polygen ${ }^{\text {Th }}$ (MVP Laboratories, Omaha, NE, USA), 55\% of Stimune (Thermo Fisher Scientific, Waltham, MA, USA) or $50 \%$ of Montanide ${ }^{\text {tux }}$ ISA 50 V2 (Seppic, Paris, France), following the manufacturers' recommendations. A control group received $100 \mu \mathrm{L}$ of PBS. To follow antibody responses, mice were bled $(100 \mu \mathrm{L})$ before immunization, and twelve days after the first and the second vaccination doses by the dorsal tail vein. Fifteen days after the second vaccination, animals were intraperitoneally challenged as described below.

\section{Bacterial challenge}

A mouse model of infection was used $(38,39)$. These studies were carried out in strict accordance with the recommendations of and approved by the University of Montreal Animal Welfare Committee guidelines and policies, including euthanasia to minimize animal suffering through the use of humane endpoints, applied throughout this study when animals were seriously affected since mortality was not an endpoint measurement. Immunized and control mice were inoculated with $4 \times 10^{7} \mathrm{CFU}$ via the intraperitoneal route. Health and behavior were monitored at least thrice daily until $72 \mathrm{~h}$ post-infection and twice thereafter until the end of the experiment (14 days post-infection) for the development of clinical signs of sepsis, such as depression, swollen eyes, rough hair coat, prostration, and lethargy. Mice were immediately euthanized upon reaching endpoint criteria using $\mathrm{CO} 2$ followed by cervical dislocation. No mice died before meeting endpoint criteria and all surviving mice were euthanized as described above at the end of the experiment ( 14 days p.i.).

\section{Antibody titration}

Polysorp immunoplates (Nunc, Roskilde, Denmark) were coated with a solution of $0.3 \mu \mathrm{g} / \mathrm{mL}$ rSsEno ou rDPPIV in carbonate buffer $(0.1 \mathrm{M}$ [pH 9.6] at $100 \mu \mathrm{L} /$ well $)$ for 1 $\mathrm{h}$ at $37^{\circ} \mathrm{C}$. After washing with PBS containing $0.05 \%$ Tween 20 (PBS-T), mouse sera were serially diluted (2fold) in PBS-T and incubated for $90 \mathrm{~min}$ at room temperature (RT). Plates were then washed in PBS-T and incubated with peroxidase-conjugated goat antimouse total Ig (IgG plus IgM) (Jackson ImmunoResearch), IgM, IgG1, IgG2b, IgG2c or IgG3 (Southern Biotech, Birmingham, AL, USA) antibodies for $1 \mathrm{~h}$ at $\mathrm{RT}$. Isotype IgG2a was not measured as it is considered to be homologous to IgG2c in mice (41). After washing, plates were developed with $3,3^{\prime}, 5,5^{\prime}$-tetramethylbenzidine $(\mathrm{TMB})$ substrate and the enzyme reaction was stopped by addition of $1 \mathrm{M} \mathrm{H}_{2} \mathrm{SO}_{4}$. Absorbance was read at $450 \mathrm{~nm}$ with an ELISA plate reader. The reciprocal of the last serum dilution that resulted in an optical density at $450 \mathrm{~nm}\left(\mathrm{OD}_{450}\right)$ of $\leq 0.1$ (cutoff) was considered the titer of that serum.

\section{Statistics}

Data are expressed as mean \pm standard error of the mean (SEM). Significant differences in between groups were determined using the t-test and one-way ANOVA, where appropriate. For in vivo virulence experiments, survival was analyzed using the LogRank test. A $p<0.05$ was considered statistically significant.

\section{Abbreviations \\ CPS: capsular polysaccharide; DPPIV: dipeptidyl peptidase IV; HRP: horseradish peroxidase; PBS: phosphate-buffered saline; rDPPIV: recombinant dipeptidyl peptidase IV; rSsEno: recombinant enolase; SEM: Standard error of the mean; SsEno: enolase; TBS: Tris-buffered saline; THB: Todd-Hewitt broth; \\ TMB: 3,3',5,5'-tetramethylbenzidine}

\section{Acknowledgments \\ The authors would like to thank S. Lacouture, P. Lemire and C. Calzas (University of Montreal) for invaluable technical assistance.}

\section{Author's contribution \\ Conception of the work: AD, DG, JH, EN, MG; laboratory techniques: AD, LM, $D G, J P A$, acquisition, analysis and interpretation of data: $A D, L M, D G, J P A, J H$, EN, MG; preparation of the manuscript: AD, MG. All authors have read, corrected and approved this manuscript.}

\section{Funding}

The design of the study, analysis and interpretation of data as well as the writing was funded by the Natural Sciences and Engineering Research Council of Canada (NSERC-RDC grant 468787-14 with Prevtec Microbia Inc.) to $M G, J H, D G$, and EN. JPA is the recipient of an Alexander Graham Bell Graduate Scholarship - Doctoral Program from NSERC.

\section{Availability of data and materials}

The data and materials not presented in this manuscript are available from the corresponding author upon request. 


\section{Ethics approval and consent to participate}

All experiments involving mice were conducted in accordance with the guidelines and policies of the Canadian Council on Animal Care and the principles set forth in the Guide for the Care and Use of Laboratory Animals by the Animal Welfare Committee of the University of Montreal (RECH-1748).

\section{Consent for publication}

Not applicable.

\section{Competing interests}

No competing interest to declare.

\section{Author details}

${ }^{1} G$ roupe de recherche sur les maladies infectieuses en production animale (GREMIP), Faculty of Veterinary Medicine, University of Montreal, 3200 Sicotte St.., Saint-Hyacinthe, QC J2S 2M2, Canada. ${ }^{2}$ Swine and Poultry Infectious Diseases Research Center (CRIPA), Montreal, Quebec, Canada. ${ }^{3}$ Oral Ecology Research Group (GREB), Faculty of Dentistry, Laval University, Quebec City, Quebec, Canada. ${ }^{4}$ Prevtec Microbia Inc. 3395 Casavant W. Blvd, Saint-Hyacinthe, QC J2S OB8, Canada.

\section{Received: 28 July 2019 Accepted: 26 November 2019}

\section{Published online: 10 December 2019}

\section{References}

1. Gottschalk M, Segura M, Xu J. Streptococcus suis infections in humans: the Chinese experience and the situation in North America. Anim Health Res Rev. 2007;8(1):29-45.

2. Wertheim HF, Nghia HD, Taylor W, Schultsz C. Streptococcus suis: an emerging human pathogen. Clin Infect Dis. 2009;48(5):617-25.

3. Gottschalk M, Xu J, Calzas C, Segura M. Streptococcus suis: a new emerging or an old neglected zoonotic pathogen? Future Microbiol. 2010;5(3):371-91.

4. Okura M, Osaki M, Nomoto R, Arai S, Osawa R, Sekizaki T, et al. Current Taxonomical Situation of Streptococcus suis. Pathogens. 2016;5(3).

5. Zheng H, Qiu X, Roy D, Segura M, Du P, Xu J, et al. Genotyping and investigating capsular polysaccharide synthesis gene loci of nonserotypeable Streptococcus suis isolated from diseased pigs in Canada. Vet Res. 2017:48(1):10.

6. Goyette-Desjardins G, Auger JP, Xu J, Segura M, Gottschalk M. Streptococcus suis, an important pig pathogen and emerging zoonotic agent-an update on the worldwide distribution based on serotyping and sequence typing. Emerg Microbes Infect. 2014;3(6):e45.

7. Gottschalk M, Lacouture S. Canada: distribution of Streptococcus suis (from 2012 to 2014) and Actinobacillus pleuropneumoniae (from 2011 to 2014) serotypes isolated from diseased pigs. Can Vet J. 2015;56(10):1093-4.

8. Segura M, Calzas C, Grenier D, Gottschalk M. Initial steps of the pathogenesis of the infection caused by Streptococcus suis: fighting against nonspecific defenses. FEBS Lett. 2016;590(21):3772-99.

9. Fittipaldi N, Segura M, Grenier D, Gottschalk M. Virulence factors involved in the pathogenesis of the infection caused by the swine pathogen and zoonotic agent Streptococcus suis. Future Microbiol. 2012;7(2):259-79.

10. Segura M. Streptococcus suis vaccines: candidate antigens and progress. Expert Rev Vaccines. 2015;14(12):1587-608.

11. Hopkins D, Poljak Z, Farzan A, Friendship R. Field studies evaluating the direct, indirect, total, and overall efficacy of Streptococcus suis autogenous vaccine in nursery pigs. Can Vet J. 2019;60(4):386-90

12. Goyette-Desjardins G, Calzas C, Shiao TC, Neubauer A, Kempker J, Roy R, et al. Protection against Streptococcus suis serotype 2 infection using a capsular polysaccharide Glycoconjugate vaccine. Infect Immun. 2016;84(7): 2059-75.

13. Brockmeier SL, Loving CL, Nicholson TL, Wang J, Peters SE, Weinert L, et al. Use of Proteins Identified through a Functional Genomic Screen To Develop a Protein Subunit Vaccine That Provides Significant Protection against Virulent Streptococcus suis in Pigs. Infect Immun. 2018;86(3).

14. Feng L, Niu X, Mei W, Li W, Liu Y, Willias SP, et al. Immunogenicity and protective capacity of EF-Tu and FtsZ of Streptococcus suis serotype 2 against lethal infection. Vaccine. 2018;36(19):2581-8.

15. Fu L, Zhao J, Lin L, Zhang Q, Xu Z, Han L, et al. Characterization of IgA1 protease as a surface protective antigen of Streptococcus suis serotype 2. Microbes Infect. 2016;18(4):285-9.
16. Wang Y, Yi L, Sun LY, Liu YC, Wen WY, Li XK, et al. Identification and characterization of a Streptococcus suis immunogenic ornithine carbamoytransferase involved in bacterial adherence. J Microbiol Immunol Infect. 2018.

17. Hsueh KJ, Cheng LT, Lee JW, Chung YC, Chung WB, Chu CY. Immunization with Streptococcus suis bacterin plus recombinant Sao protein in sows conveys passive immunity to their piglets. BMC Vet Res. 2017;13(1):15.

18. Li Y, Gottschalk M, Esgleas M, Lacouture S, Dubreuil JD, Willson P, et al. Immunization with recombinant Sao protein confers protection against Streptococcus suis infection. Clin Vaccine Immunol. 2007;14(8):937-43.

19. Li Y, Martinez G, Gottschalk M, Lacouture S, Willson P, Dubreuil JD, et al. Identification of a surface protein of Streptococcus suis and evaluation of its immunogenic and protective capacity in pigs. Infect Immun. 2006;74(1):305-12.

20. Auger JP, Chuzeville S, Roy D, Mathieu-Denoncourt A, Xu J, Grenier D, et al. The bias of experimental design, including strain background, in the determination of critical Streptococcus suis serotype 2 virulence factors. PLoS One. 2017;12(7):e0181920.

21. Esgleas M, Li Y, Hancock MA, Harel J, Dubreuil JD, Gottschalk M. Isolation and characterization of alpha-enolase, a novel fibronectin-binding protein from Streptococcus suis. Microbiology. 2008;154(Pt 9):2668-79.

22. Sun Y, Li N, Zhang J, Liu H, Liu J, Xia X, et al. Enolase of Streptococcus Suis serotype 2 enhances blood-brain barrier permeability by inducing IL-8 release. Inflammation. 2016;39(2):718-26.

23. Esgleas M, Dominguez-Punaro Mde L, Li Y, Harel J, Dubreuil JD, Gottschalk M. Immunization with SsEno fails to protect mice against challenge with Streptococcus suis serotype 2. FEMS Microbiol Lett. 2009;294(1):82-8.

24. Feng Y, Pan X, Sun W, Wang C, Zhang H, Li X, et al. Streptococcus suis enolase functions as a protective antigen displayed on the bacterial cell surface. J Infect Dis. 2009;200(10):1583-92.

25. Gottschalk M. Streptococcosis. In: KL ZJJ, Ramirez ASK, Stevenson GW, editors. Diseases of Swine. 10Th ed. Hoboken, NJ: WileyBlackwell; 2012. p. $841-55$

26. Seitz M, Valentin-Weigand P, Willenborg J. Use of antibiotics and antimicrobial resistance in veterinary medicine as exemplified by the swine pathogen Streptococcus suis. Curr Top Microbiol Immunol. 2016:398:103-21.

27. Dinis M, Tavares D, Veiga-Malta I, Fonseca AJ, Andrade EB, Trigo G, et al. Oral therapeutic vaccination with Streptococcus sobrinus recombinant enolase confers protection against dental caries in rats. J Infect Dis. 2009; 199(1):116-23.

28. Membrebe JD, Yoon NK, Hong M, Lee J, Lee H, Park K, et al. Protective efficacy of Streptococcus iniae derived enolase against streptococcal infection in a zebrafish model. Vet Immunol Immunopathol. 2016;170:25-9.

29. Wang J, Wang K, Chen D, Geng Y, Huang X, He Y, et al. Cloning and characterization of surface-localized alpha-Enolase of Streptococcus iniae, an effective protective antigen in mice. Int J Mol Sci. 2015;16(7):14490-510.

30. Zhang A, Chen B, Mu X, Li R, Zheng P, Zhao Y, et al. Identification and characterization of a novel protective antigen, Enolase of Streptococcus suis serotype 2. Vaccine. 2009;27(9):1348-53.

31. Trunova GV, Makarova OV, Diatroptov ME, Bogdanova IM, Mikchailova LP, Abdulaeva SO. Morphofunctional characteristic of the immune system in BALB/C and C57BL/6 mice. Bull Exp Biol Med. 2011;151(1):99-102.

32. Mills CD, Kincaid K, Alt JM, Heilman MJ, Hill AM. M-1/M-2 macrophages and the Th1/Th2 paradigm. J Immunol. 2000;164(12):6166-73.

33. Im EJ, Hanke T. Short communication: preclinical evaluation of candidate HIV type 1 vaccines in inbred strains and an outbred stock of mice. AIDS Res Hum Retrovir. 2007;23(7):857-62.

34. Jobin MC, Martinez G, Motard J, Gottschalk M, Grenier D. Cloning, purification, and enzymatic properties of dipeptidyl peptidase IV from the swine pathogen Streptococcus suis. J Bacteriol. 2005;187(2):795-9.

35. Ge J, Feng $Y$, Ji H, Zhang H, Zheng F, Wang C, et al. Inactivation of dipeptidyl peptidase IV attenuates the virulence of Streptococcus suis serotype 2 that causes streptococcal toxic shock syndrome. Curr Microbiol. 2009;59(3):248-55.

36. Yagishita H, Kumagai Y, Konishi K, Takahashi Y, Aoba T, Yoshikawa M. Histopathological studies on virulence of dipeptidyl aminopeptidase IV (DPPIV) of Porphyromonas gingivalis in a mouse abscess model: use of a DPPIV-deficient mutant. Infect Immun. 2001;69(11):7159-61.

37. Segura M, Fittipaldi N, Calzas C, Gottschalk M. Critical Streptococcus suis virulence factors: are they all really critical? Trends Microbiol. 2017;25(7):585-99. 
38. Dominguez-Punaro MC, Segura M, Plante MM, Lacouture S, Rivest S, Gottschalk M. Streptococcus suis serotype 2, an important swine and human pathogen, induces strong systemic and cerebral inflammatory responses in a mouse model of infection. J Immunol. 2007:179(3):1842-54.

39. Auger JP, Fittipaldi N, Benoit-Biancamano MO, Segura M, Virulence GM. Studies of Different Sequence Types and Geographical Origins of Streptococcus suis Serotype 2 in a Mouse Model of Infection. Pathogens. 2016;5(3) Available from: http://www.ncbi.nlm.nih.gov/pubmed/27409640

40. Chaffin DO, Mentele LM, Rubens CE. Sialylation of group B streptococcal capsular polysaccharide is mediated by cpsK and is required for optimal capsule polymerization and expression. J Bacteriol. 2005;187(13):4615-26.

41. Collins AM. IgG subclass co-expression brings harmony to the quartet model of murine lgG function. Immunol Cell Biol. 2016;94(10):949-54.

42. Kobisch M, Gottschalk M, Morvan P, Cariolet R, Bénévent G, Joly JP. Experimental infection of SPF piglets with Streptococcus suis serotype 2. J Rech Porcine. 1995;27:97-102.

\section{Publisher's Note}

Springer Nature remains neutral with regard to jurisdictional claims in published maps and institutional affiliations.

Ready to submit your research? Choose BMC and benefit from:

- fast, convenient online submission

- thorough peer review by experienced researchers in your field

- rapid publication on acceptance

- support for research data, including large and complex data types

- gold Open Access which fosters wider collaboration and increased citations

- maximum visibility for your research: over $100 \mathrm{M}$ website views per year

At BMC, research is always in progress.

Learn more biomedcentral.com/submissions 